\title{
Bacteriocin release protein mediated secretory expression of recombinant chalcone synthase in Escherichia coli
}

\begin{abstract}
Flavonoids are secondary metabolites synthesized by plants shown to exhibit health benefits such as anti-inflammatory, antioxidant, and anti-tumor effects. Thus, due to the importance of this compound, several enzymes involved in the flavonoid pathway have been cloned and characterized in Escherichia coli. However, the formation of inclusion bodies has become a major disadvantage of this approach. As an alternative, chalcone synthase from Physcomitrella patens was secreted into the medium using a bacteriocin release protein expression vector. Secretion of $\mathrm{P}$. patens chalcone synthase into the culture media was achieved by co-expression with a psW1 plasmid encoding bacteriocin release protein in $\mathrm{E}$. coli Tuner (DE3) plysS. The optimized conditions, which include the incubation of cells for $20 \mathrm{~h}$ with $40 \mathrm{ng} / \mathrm{ml}$ mitomycin C at OD600 induction time of 0.5 was found to be the best condition for chalcone synthase secretion.
\end{abstract}

Keyword: Bacteriocin release protein; Chalcone synthase; Extracellular expression 